\title{
Neuropsychological, Behavioral, and Quality of Life Outcomes in Children and Adolescents with Sickle Cell Disease Treated with Nonmyeloablative Matched Sibling Donor Hematopoietic Cell Transplantation: A case series.
}

Taryn Fay-McClymont ${ }^{1}$, Dania Monagel $^{2}$, GURPREET SINGH ${ }^{1}$, Fiona Schulte ${ }^{3}$, Brian Brooks $^{1}$, William MacAllister ${ }^{1}$, Naddley Desire ${ }^{4}$, Aleksandra Mineyko ${ }^{3}$, Marsha Vasserman $^{1}$, Michael Leaker ${ }^{1}$, Tony Truong ${ }^{1}$, Ravi Shah ${ }^{1}$, Victor Lewis ${ }^{5}$, Keith Yeates ${ }^{6}$, and Gregory Guilcher ${ }^{1}$

${ }^{1}$ Alberta Children's Hospital

${ }^{2}$ King Saud bin Abdulaziz University for Health Sciences

${ }^{3}$ University of Calgary Cumming School of Medicine

${ }^{4}$ The Hospital for Sick Children

${ }^{5}$ Alberta Childrens Hosptial

${ }^{6}$ Alberta Children's Hospital Research Institute for Child and Maternal Health

January 29, 2022

\begin{abstract}
Background/Objectives. Despite advances in the treatment of sickle cell disease (SCD), cerebrovascular and cognitive consequences can be lifelong. Hematopoietic cell transplantation (HCT) is an established curative therapy and recent studies have demonstrated efficacy with reduced toxicity nonmyeloblative (NMA) regimens, but little is known about neuropsychological outcomes. The objective of this study was to describe neuropsychological, behavioral, and quality of life outcomes with medical correlates in children with SCD who received an NMA matched sibling donor (MSD) HCT. Design/Methods. This retrospective cohort analysis of nine patients with hemoglobin SS SCD underwent MSD HCT using the National Institutes of Health (NIH) NMA protocol. Results. Mean full scale intellectual functioning (FSIQ) was average pre-HCT (FSIQ=92.1, SD 9.0; n=8) and 2 years post-HCT (mean FSIQ=96.6; SD 11.1; N=9). Neuropsychological functioning was largely average across all cognitive domains. Moderate improvements were seen in processing speed and verbal memory (Cohen's d=0.50-0.57) post-HCT, and declines occurred in measures of attention and fine motor speed and dexterity (Cohen's d=0.70-0.81). Parents reported improved quality of life (Cohen's d=0.91), less impact of SCD on their family, and less worry about their child's future (Cohen's d=1.44). Exploratory analysis showed relationships between pre-HCT hemoglobin $(\mathrm{r}=0.74, \mathrm{p}<0.05)$ and creatinine $(\mathrm{r}=-0.75, \mathrm{p}<0.01)$ with cognitive functioning, and a positive relationship between processing speed and time post-HCT ( $\mathrm{r}=0.73$ ). Conclusion. Neuropsychological functioning in a sample of children and adolescents treated identically with NMA MSD HCT remained stable or improved in most cognitive domains, and improvements in quality of life and family functioning were observed.
\end{abstract}

\section{INTRODUCTION}

Sickle cell disease (SCD) is an autosomal recessive disorder that causes irreversible damage to multiple organs due to chronic hemolytic anemia and recurrent vasoocclusive episodes and systemic vasculopathy ${ }^{1}$. Hematopoietic cell transplantation (HCT) is a potentially curative therapy with relatively stable cerebrovascular disease described post-HCT, particularly in those who receive reduced intensive conditioning ${ }^{2,3}$. Rates 
of graft-versus-host disease (GVHD) are lowest for those recipients with a matched sibling donor (MSD) ${ }^{4}$. High rates of survival (91-100\%) have been described in children and adolescents who undergo myeloablative conditioning (MAC) HCT with a matched related donor ${ }^{3,4}$. Despite these successes, acute and late toxicities remain a concern, particularly in children who do not have a donor option available ${ }^{4,5}$. Notably, MAC conditioning and calcineurin inhibitors come with increased risks of neurotoxicity ${ }^{6}$. Despite the fact that over 1000 transplants have been performed for SCD, long-term outcomes other than survival and graft failure remain poorly described ${ }^{1,3}$.

The primary risk factors for cognitive impairment in childhood SCD include chronic anemia, systemic ischemia, silent cerebral infarcts, and overt strokes ${ }^{7,8}$. While the incidence of overt stroke is decreasing due to primary stroke prevention strategies such as Transcranial Doppler (TCD) screening, transfusion therapy, and hydroxyurea, cerebrovascular compromise is still common and up to $40 \%$ of children and adolescents have MRI abnormalities, primarily in the frontal lobe ${ }^{9,10}$. Neuropsychological deficits in children with SCD include aspects of cognition implicated in the frontal-subcortical networks, such as attention, executive functioning, working memory, and processing speed, with up to $25 \%$ of children meeting diagnostic criteria for attention deficit hyperactivity disorder (ADHD) by adolescence, compared to about $7 \%$ in the general population ${ }^{7,11-17}$. Additionally, children with SCD generally show lower levels of intellectual and academic functioning even without evidence of cerebral infarction, and declines in intellectual functioning over time have been shown in children with infarcts ${ }^{12,18}$. Neurodevelopmental delays are also commonly found in pre-school children with $\mathrm{SCD}^{19}$. A number of studies have found that predictors of neuropsychological impairment outside of stroke include low hemoglobin and hematocrit, elevated TCD velocity, reduced white matter integrity, older age (younger age confers better outcome), lower socioeconomic status, financial stress, and poor access to medical care ${ }^{7,8,16,20}$. Disparities in the treatment of pain in SCD, individual and systemic racism in healthcare, mistrust of healthcare providers due to historical medical exploitation, and the stigma attached to SCD have also been shown to impact the care and lives of marginalized black children and their families ${ }^{21,22}$.

Little is known about post-HCT neuropsychological functioning, particularly in children and adolescents without cognitive deficits or evidence of stroke before transplant. A small number of studies describe intellectual functioning post-HCT, but the challenge in interpreting these outcomes is that the clinical manifestations that ultimately make a child eligible for HCT- such as stroke, poorer cognitive functioning, or elevated TCD velocities - may also yield negative neurocognitive outcomes.

Only five small cohort studies have described neurocognitive functioning post-HCT, with results demonstrating largely stable MRIs post-HCT, and stable or improved intellectual functioning and processing speed, despite many children having evidence of overt or silent stroke pre-HCT ${ }^{23-27}$.

Given the relative dearth of evidence regarding all aspects of functioning post-HCT in children, this study sought to describe neuropsychological, behavioral and quality of life outcomes with medical correlates in a small sample of children with SCD without baseline cerebrovascular disease who underwent an NMA MSD HCT.

\section{METHODS}

\section{Design}

This study involved a retrospective cohort analysis of all patients with SCD who completed a MSD HCT at the Alberta Children's Hospital using the National Institutes of Health (NIH) NMA protocol. The study includes patients who were seen pre-HCT for a clinical neuropsychological assessment, and again approximately two years post-HCT. The study was approved by the Conjoint Health Research Ethics Board at the University of Calgary. All patients and/or guardians signed institutional review board-approved informed consent or assent documents.

\section{Participants}

All included patients met institutional eligibility for $\mathrm{HCT}$, including age-appropriate cardiac, respiratory, 
hepatic, and renal evaluations ${ }^{2}$. Siblings were 10/10 Human Leukocyte Antigens (HLA) matched and underwent comprehensive medical and psychosocial clearance processes ${ }^{28}$; donors could have HbAA or HbAS. All patients received identical treatment (NMA conditioning regimen of alemtuzumab, single fraction of $300 \mathrm{cGy}$ total body irradiation, GVHD prophylaxis with prolonged sirolimus), and were taking hydroxyurea until the time of transplant.

All children who received an MSD HCT using the NIH protocol with long-term follow-up at the Alberta Children's Hospital were offered pre- and post-HCT clinical neuropsychological assessments as part of standard routine care and free of charge to the patient in the Canadian healthcare system.

A total of 8 patients had pre and post-HCT neuropsychological assessments. One patient was too young (3 years old) to participate in the pre-HCT assessment but was included in the post-HCT assessment. Two patients declined post-HCT assessment despite repeated attempts to schedule them. One of these patients had evidence of stroke pre-HCT. Detailed medical chart reviews were completed, and medical, demographic and psychosocial information was also collected. Specific medical details and outcomes for the larger cohort at our institution were published previously ${ }^{2}$.

\section{Measures}

Demographics. Demographic variables included date of birth, age, sex, ethnicity, parental education, and primary language.

Medical variables . Medical variables included HCT date; pre- and post-HCT TCD results (highest proximal middle cerebral artery velocity); brain MRI results (where available); pre-HCT SCD medications and treatments, including hydroxyurea and chronic transfusions; Hb levels pre-HCT, at 6 months and 1-year post-HCT; baseline ferritin, creatinine, glomerular filtration rate (GFR); chimerism results; and post-HCT HbS level.

Neuropsychological Tests. Standardized neuropsychological measures with strong psychometric properties were used to assess intellectual functioning, processing speed, and working memory (i.e., Wechsler intelligence scales including: WPPSI-IV, WAIS-IV, WISC-IV, WISC-V; estimated IQ used in some patients), sustained attention (i.e., TOVA, CPT-2), executive functioning (i.e., NEPSY-II Inhibition Test), verbal and visual memory (i.e., ChAMP [lists/objects], CVLT-C, or CVMT), academic skills (i.e., math, reading, and spelling; WIAT-III), and fine motor skills (i.e., Purdue Pegboard). All patients obtained scores above accepted cut-offs on performance validity tests.

Parent/Child Questionnaires . Questionnaires administered included parent-rated measures of adaptive (i.e., SIB-R, ABAS-3), behavioral (i.e., CBCL, BASC-2, BASC-3), and executive functioning (i.e., BRIEF, BRIEF-2, BRIEF-A), as well as attention (i.e., ADHD-IV, ADHD-V) and quality of life (i.e., PedsQL Parent). Patients were also asked to rate their quality of life (i.e., PedsQL Child).

Statistical Analysis . Given the small number of patients, and the large amount of data from the preand post-HCT assessments, the primary aims were to describe the sample and conduct exploratory analysis. Relationships between neuropsychological outcomes and medical and demographic data were explored using Pearson bivariate correlations. Significant relationships were further examined using scatterplots. Paired sample t-tests were performed to examine cognitive changes across time and Cohen's d were calculated to examine the magnitude of change, given the small sample size. When examining change post-HCT, significant change was defined as at least one standard deviation (i.e., standard score change of 15 points or more ${ }^{29,30}$ ). Due to differences in measures across ages, traditional methods of assessing change (e.g., reliable change indices) were not able to be calculated. SPSS version 19 was used for all analyses.

\section{RESULTS}

Demographics. Patient characteristics are summarized in Table 1. All children were diagnosed with hemoglobin SS (HbSS) sickle cell disease. Eight children were identified by their parents as Black, and 1 child as Middle Eastern (categories based on the Canadian Institute for Health Information race-based and 
indigenous identity data collection and health reporting standards). While all families identified as English speaking, 3 families were bilingual (French/English and Arabic/English). All were two-parent households. Parents were well-educated, with $7 / 9$ mothers and 5/9 fathers having university degrees (2 MDs, 3 Masters level); all parents had a minimum of a high school education. Median patient age was 11.5 years (Mean=11.1; SD 3.6) at the pre-HCT assessment and 12.5 years (Mean=13; SD 4.2) at the post-HCT assessment. Six of nine patients $(66.7 \%)$ were female.

Medical Correlates. Two children were received chronic transfusions pre-HCT. One child was receiving chronic transfusions for 18 months pre-HCT for borderline elevated TCD findings. The other child had recurrent vasoocclusive crises and episodes of acute chest syndrome. Three children had a pre-HCT brain MRI and both were reported as 'normal', meaning no definitive evidence of stroke or neurovascular disease. Most children $(n=6 ; 67 \%)$ were taking hydroxyurea at time of HCT referral; those that were not had a minimum of 4-6 months of exposure before HCT (range 4-132 months). Median pre-HCT levels were $202 \mathrm{ug} / \mathrm{L}$ for ferritin (normal range $=10-110 \mathrm{ug} / \mathrm{L}$ ) and $29 \mathrm{umol} / \mathrm{L}$ for creatinine (normal range $=20-75$ umol/L). Median GFR values pre-HCT were $160 \mathrm{~mL} / \mathrm{min} / 1.73 \mathrm{~m}^{2}$ ([?]90 is normal or high). All pre-HCT and post-HCT TCD results were within normal ranges11TCD velocities used in the current study included the maximum value from the right and left proximal middle cerebral arteries. Velocities of less than $170 \mathrm{~cm} / \mathrm{s}$ were considered normal, those between 170 and $200 \mathrm{~cm} / \mathrm{s}$ were conditional, and those exceeding $200 \mathrm{~cm} / \mathrm{s}$ were classified as abnormal. (pre-HCT median maximum value $=125 \mathrm{~cm} / \mathrm{s} ;$ post-HCT=91 cm $/ \mathrm{s}$ ). PostHCT TCD results were not available for two patients. Median transfusion hemoglobin $(\mathrm{Hb}$ ) level (normal values=110-157 g/L) was $91 \mathrm{~g} / \mathrm{L}$ pre-HCT, $133 \mathrm{~g} / \mathrm{L}$ at 6 months post-HCT, and $135 \mathrm{~g} / \mathrm{L}$ at 1-year post-HCT. The median $\mathrm{Hb}$ change scores was -42 (range -17 to -51 ). The median percent donor T-cell chimerism by 24 months post-HCT was $77 \%$ donor, and the myeloid chimerism was $100 \%$ donor for all children but one (97\%). No patient developed clinical evidence of sickle cell disease by two years post-HCT, and all had $\mathrm{Hb}$ S levels less than 50\%. No patient had post-HCT neurological complications (i.e., hemorrhage, stroke, encephalopathy, or seizure) and none developed GVHD.

Neuropsychological Outcomes. Mean FSIQ at the pre-HCT assessment was within the average range (i.e., between the $25-74^{\text {th }}$ percentile, or a standard score of $90-109^{31}$; FSIQ=92.1, SD 9.0; median=92.5). One child who had below average FSIQ at the pre-HCT assessment, had low average FSIQ at the post-HCT assessment (increase of 12 standard score points). As per Table 2 and Figure 1, most pre-HCT neuropsychological results were within the average range, with group means for each domain in the average range. The lowest mean pre-HCT mean score was in sustained attention (Mean=90.5, SD 18.9; median=98.5).

Post-HCT Change. Post-HCT neuropsychological assessments were conducted 2.27 years after HCT (SD .72 ), on average. Mean FSIQ remained in the average range (mean FSIQ=96.6; SD 11.1; median=95). Paired sample t-tests were not significant for any pre/post-HCT comparisons given the small sample size, but effect sizes ranged from moderate to large overall (as per Cohen's d).

Most post-HCT results remained in the average range. Moderate effect sizes were found for improvements in processing speed (mean pre-HCT PSI=95.1, SD 5.8 [median=95.5]; post-HCT PSI=101.6, SD 11.4 [median=100]; Cohen's $d=0.57$ ) and verbal memory (mean pre-HCT verbal memory=104.8, SD 8.0 $[$ median $=103]$; post-HCT verbal memory=111.2, SD $12.5[$ median $=115]$; Cohen's $d=0.50)$. Small to medium effect sizes were found for improvements in executive functioning post-HCT (mean pre-HCT inhibition=91.3, SD 10.6 [median=92.5]; post-HCT inhibition=95.8, SD 16.0 [median=95]; Cohen's $d=0.33$ ). Two children demonstrated significant declines in attention post-HCT (Fig. 1), with a moderate to large effect size found at the group level (i.e., pre-HCT sustained attention=90.5, SD 18.9 [median=98.5]; post-HCT attention=78.4, SD 25.4 [median=83]; Cohen's $d=-0.70)$. Fine motor speed and dexterity declines were observed in four children post-HCT, with a large effect size at the group level (mean pre-HCT fine motor=91.3, SD 10.7 $[$ median=89]; post-HCT fine motor=75.9, SD 20.6 [median=70]; Cohen's $d=-0.81$ ).

Some neuropsychological outcomes were not included in Table 2 because less data were captured at the pre-HCT assessment because of differences in neuropsychological tests. Average WMI was 105.8 (SD 14.5; median=103; $\mathrm{n}=5$ ) pre-HCT and 96.9 (SD 17.4; median=100; $\mathrm{n}=8$ ) post-HCT. Six children had single word 
reading scores from the WIAT-III, with means of 100.7 (SD 19.3; median=101) pre-HCT and 100.2 (SD 16.7; median=106; n=9) post-HCT. Five children had spelling and math (numerical operations) results from pre-HCT, with mean scores in the average range (Spelling=104.0, SD 14.2 [median=100]; Math=104.2, SD 22.4 [median=112]). At post-HCT, all 9 children had spelling and math results in the average range (mean spelling=103.8, SD 18.5, median=108; math=101.8, SD 18.8, median=105).

Parent-Rated Outcomes and Quality of Life. Not all questionnaires were completed because of differences in assessment protocols or unavailability of parent questionnaires. As seen in Table 3, no difficulties with attention, executive functioning, or adaptive and behavioral functioning were reported by parents preor post-HCT. Significant pre- and post-HCT attention difficulties were reported in one child, who was diagnosed with Attention Deficit/Hyperactivity Disorder (ADHD, Inattentive type) post-HCT. New, mild attention difficulties were reported in one child post-HCT, and slightly more executive functioning difficulties were reported in another child post-HCT, but these problems did not reach clinical cut-offs. Parents endorsed slightly less difficulties with executive functioning in two children post-HCT, and three children were rated as having higher daily adaptive functioning post-HCT. No clinically-elevated behavioral or emotional problems were reported in any child at any time point, but slightly more difficulties were reported post-HCT in two children. Parent rated quality of life improved in 4/9 children post-HCT, with a large effect size (pre-HCT mean PedsQL=77.9, SD 16.4 [median=76]; post-HCT PedsQL=89.7, SD 8.0 [median=87]; Cohen's $d=0.91$ ). In paired sample t-tests, this result was significant, $\mathrm{t}(6)=-2.69, \mathrm{p}=0.04$. The Family Impact Module of the PedsQL was not included in Table 3 because only five parents completed this measure pre-HCT, but the overall post-HCT mean score was significantly higher, with large effect size (i.e., pre-HCT mean=79.5, SD 15.1 [median=81.5]; post-HCT mean=96, SD 5.8 [median=100]; Cohen's $d=1.44$ ). Parents reported less impact of their child's illness on their family, and less worry about their medical treatments and future. While two children rated their overall quality of life as improved at the post-HCT assessment (Table 3), the mean total score did not improve significantly (pre-HCT child-rated PedsQL=65.9, SD 15.4 [median=63.5]; post-HCT child-rated PedsQL=71.5, SD 19.7 [median=76], Cohen's $d=0.32$ ).

Associations between Demographic, Medical Factors, and Neuropsychological Results. Higher pre-HCT creatinine levels were associated with lower pre-HCT visual memory $(\mathrm{r}=-0.745, \mathrm{p}<.01)$. Additionally, pre-HCT GFR was associated with pre-HCT sustained attention $(\mathrm{r}=0.793, \mathrm{p}<.05)$, with lower GFR associated with worse attention scores. Lower pre-HCT hemoglobin level was significantly correlated with post-HCT FSIQ, such that children with lower hemoglobin levels pre-HCT had lower FSIQ post-HCT $(\mathrm{r}=0.744, \mathrm{p}<.05)$. Change in Hb score from pre-HCT to 6 months post-HCT was not significantly related to FSIQ post-HCT, although children with greater increases in Hb post-HCT tended to have lower FSIQ $(\mathrm{r}=-0.648, \mathrm{p}=.059)$. A significant correlation was found between time from HCT to the neuropsychological assessment and post-HCT processing speed $(\mathrm{r}=0.729, \mathrm{p}<.01)$, with higher processing speed in children who were tested at a longer interval post-HCT (Fig. 2). Finally, lower post-HCT max TCD was associated with better child rated quality of life $(\mathrm{r}=-0.894, \mathrm{p}<.05)$.

\section{DISCUSSION}

This study examined neuropsychological functioning in a sample of children and adolescents with HbSS disease and no overt history of stroke who were treated identically with an NMA MSD HCT. The medical outcomes of this cohort have been published previously, demonstrating $100 \%$ survival, no sickling crises, and no graft versus host disease or neurological complications ${ }^{2}$. These results demonstrate mostly stable-toimproved neuropsychological outcomes post-HCT, in a cohort with no overt baseline neurovascular disease and high levels of parental education, with improvements in several important areas of cognitive functioning and quality of life.

While our sample size is small and our analyses rely on retrospective data, these findings are consistent with several previously published studies on HCT in children, most notably replicating the stability of intellectual functioning and improvements in processing speed, verbal memory, and executive functioning post-HCT ${ }^{23,26}$. The more common finding in young people with SCD not treated with HCT is a slow decline in cognition over time ${ }^{18}$. Younger age at time of HCT was not related to improved cognitive outcomes as has been shown 
in previous research, but our capacity to find significant associations was undoubtedly hampered by the small sample size and the need for longer follow-up. Given the natural history of SCD and neurocognitive decline, stable cognitive function can be viewed as a positive outcome.

More difficulties were seen in neuropsychological measures of attention post-HCT, but this may also reflect the increasing age of children in the sample, and thus increased expectations for attention skills. Interestingly, attention difficulties were not reflected in parent questionnaires, with stable attention seen in 7/9 patients. Fine motor speed and dexterity was the primary area of new difficulty post-HCT, with $5 / 9$ children demonstrating deficits (standard score [?]70), and four children demonstrating declines of greater than a standard deviation. Difficulties with fine motor speed and dexterity are common in children with $\mathrm{SCD}^{19,32}$, but these increased difficulties might be transient and resolve with time. Further studies of larger samples of children and adolescents post-HCT are needed to determine if this is the case.

Questionnaire results showed some improvements in parent-rated executive and adaptive functioning, and more substantial improvements in parent-rated quality of life for the children post-HCT. Parents also reported reduced impact of their child's illness on their family functioning, and less worry about their medical treatments and future. Quality of life as rated by children improved slightly, but less so as compared to their parents' ratings. A recent systematic review of quality of life after stem cell transplant in adults ${ }^{33}$ demonstrated large positive quality of life improvements reported by patients post-HCT. Further research is needed with larger sample sizes to examine the differences between parents' and patients' perspectives post-HCT.

A few significant associations were found between medical variables and outcomes, with TCD, creatinine, GFR, and hemoglobin being related to cognitive functioning. Most notably, lower hemoglobin pre-transplant was associated with lower FSIQ post-HCT. Interestingly, those children that showed the largest change in hemoglobin post-HCT had a trend towards a lower post-HCT FSIQ, suggesting that correcting Hb did not necessarily improve cognitive functioning. A recent meta-analysis ${ }^{8}$ demonstrated a positive relationship between hemoglobin and full scale intellectual functioning, but few studies have examined the relationship between pre-HCT medical variables and post-HCT cognitive functioning in children with SCD. Our analysis was exploratory and the correlational nature of these findings precludes causal inference, but these variables should be examined in larger samples over time.

This study has several limitations including small sample size, lack of baseline MRI testing for all patients due to institutional practice at the time (resulting in a possible underestimate of silent cerebral infarcts), and possible selection bias as two patients declined a follow-up visit. One of these patients had evidence of stroke pre-HCT and we may have found more cognitive difficulties post-HCT if this patient were included. This study did not examine the impact of racial bias and stigma on cognitive, behavioral and quality of life outcomes post-HCT, but these considerations should be integrated into future research with SCD given the marked impact of individual, structural, and societal racism on health outcomes ${ }^{21,22}$. This was a relatively unique sample of patients with SCD a) whose parents were highly educated, b) for whom the cost of medical care was publicly funded, c) who did not have baseline overt cerebrovascular disease pre-HCT, and d) who had uniform HCT therapy. These limitations might also be viewed as strengths, in that outcomes were assessed in children and adolescents undergoing HCT under more ideal circumstances and yet still showed evidence of neuropsychological benefit from HCT. This study was also retrospective, which also introduced some variability into the battery of tests and questionnaires administered at each time point.

There have been recent initiatives that may help to fill some of the knowledge gaps identified by this study, including a clinical trial (https://clinicaltrials.gov/ct2/show/NCT04018937) and prospective observational study (Project Sickle Cure) led by the Sickle Transplant Advocacy and Research Alliance (STAR) These studies aim to determine the potential protective effect of early HCT on quality of life and neurocognitive function in children, as well as to study these domains in a larger cohort who receive HCT at different times and with different HCT regimens. Consensus recommendations for future clinical trial end points for patients with SCD have also been developed ${ }^{34}$.

Despite these limitations, this small case series adds to the literature that supports the safety and potential 
benefits of the NIH NMA MSD HCT protocol, with positive results showing stabilization or improvement in cognitive outcomes, improved quality of life, and no new emotional or behavioral problems. These findings lend support to early intervention with curative therapy for children and adolescents with SCD and an MSD, and indicate excellent post-HCT outcomes overall. Future research with larger sample sizes and serial long-term follow-up is needed.

\section{CONFLICT OF INTEREST STATEMENT}

Brian Brooks receives royalties for the sales of the Pediatric Forensic Neuropsychology textbook (2012, Oxford University Press) and three pediatric neuropsychological tests [Child and Adolescent Memory Profile (ChAMP, Sherman and Brooks, 2015, PAR Inc.), Memory Validity Profile (MVP, Sherman and Brooks, 2015, PAR Inc.), and Multidimensional Everyday Memory Ratings for Youth (MEMRY, Sherman and Brooks, 2017, PAR Inc.)]. He has previously received in-kind support (free test credits) from the publisher of the computerized cognitive test (CNS Vital Signs, Chapel Hill, North Carolina). He acknowledges salary funding from the Canadian Institutes for Health Research (CIHR) Embedded Clinician Researcher Salary Award.

Greg Guilcher is the Principal Investigator for Project Sickle Cure, a Sickle Cell Transplant Advocacy and Research Alliance (STAR) study partially funded by bluebirdbio.

No other authors have declared conflicts of interest.

\section{ACKNOWLEDGEMENTS}

The authors would like to thank the patients and families, as well as the many multidisciplinary team members in the hemoglobinopathy clinics in Calgary and Edmonton. Alberta Health Services supported this research project, and funding support to present preliminary findings at the International Neuropsychological Society research conference in 2019 was received by the $\mathrm{C}^{17}$ Education Committee and Childhood Cancer Canada.

\section{REFERENCES}

1. Shenoy S, Angelucci E, Arnold SD, et al. Current Results and Future Research Priorities in Late Effects after Hematopoietic Stem Cell Transplantation for Children with Sickle Cell Disease and Thalassemia: A Consensus Statement from the Second Pediatric Blood and Marrow Transplant Consortium Inte. In: Biology of Blood and Marrow Transplantation . ; 2017. doi:10.1016/j.bbmt.2017.01.009

2. Guilcher GMT, Monagel DA, Nettel-Aguirre A, et al. Nonmyeloablative Matched Sibling Donor Hematopoietic Cell Transplantation in Children and Adolescents with Sickle Cell Disease. Biol Blood Marrow Transplant . 2019. doi:10.1016/j.bbmt.2019.02.011

3. Gluckman E, Cappelli B, Bernaudin F, et al. Sickle cell disease: An international survey of results of HLA-identical sibling hematopoietic stem cell transplantation. Blood . 2017. doi:10.1182/blood-2016-10745711

4. Guilcher GMT, Truong TH, Saraf SL, Joseph JJ, Rondelli D, Hsieh MM. Curative therapies: Allogeneic hematopoietic cell transplantation from matched related donors using myeloablative, reduced intensity, and nonmyeloablative conditioning in sickle cell disease. Semin Hematol . 2018;55(2):87-93. doi:10.1053/j.seminhematol.2018.04.011

5. Walters MC, Patience M, Leisenring W, et al. Stable mixed hematopoietic chimerism after bone marrow transplantation for sickle cell anemia. Biol Blood Marrow Transplant . 2001;7(12):665-673. doi:10.1053/bbmt.2001.v7.pm11787529

6. Stenger EO, Shenoy S, Krishnamurti L. How I treat sickle cell disease with hematopoietic cell transplantation. Blood . 2019. doi:10.1182/blood.2019000821

7. Prussien K V., Salihu A, Abdullahi SU, et al. Associations of transcranial doppler velocity, age, and gender 
with cognitive function in children with sickle cell anemia in Nigeria. Child Neuropsychol . 2019;25(6):705720. doi: $10.1080 / 09297049.2018 .1526272$

8. Prussien K V., Siciliano RE, Ciriegio AE, et al. Correlates of Cognitive Function in Sickle Cell Disease: A Meta-Analysis. J Pediatr Psychol . 2020;45(2):145-155. doi:10.1093/jpepsy/jsz100

9. DeBaun MR, Armstrong FD, McKinstry RC, Ware RE, Vichinsky E, Kirkham FJ. Silent cerebral infarcts: A review on a prevalent and progressive cause of neurologic injury in sickle cell anemia. Blood . 2012. doi:10.1182/blood-2011-02-272682

10. Pegelow CH, Macklin EA, Moser FG, et al. Longitudinal changes in brain magnetic resonance imaging findings in children with sickle cell disease. Blood . 2002;99(8):3014-3018. doi:10.1182/blood.V99.8.3014

11. Steen RG, Miles MA, Helton KJ, et al. Cognitive Impairment in Children with Hemoglobin SS Sickle Cell Disease: Relationship to MR Imaging Findings and Hematocrit. AJNR Am J Neuroradiol . 2003;24(3):382389. http://www.ajnr.org/content/24/3/382.full. Accessed November 17, 2015.

12. Schatz J, Finke RL, Kellett JM, Kramer JH. Cognitive Functioning in Children With Sickle Cell Disease: A Meta-Analysis. J Pediatr Psychol . 2002;27(8):739-748. doi:10.1093/jpepsy/27.8.739

13. Hijmans CT, Fijnvandraat K, Grootenhuis MA, et al. Neurocognitive deficits in children with sickle cell disease: A comprehensive profile.Pediatr Blood Cancer . 2011;56(5):783-788. doi:10.1002/pbc.22879

14. Wang W, Enos L, Gallagher D, et al. Neuropsychologic performance in school-aged children with sickle cell disease: A report from the Cooperative Study of Sickle Cell Disease. J Pediatr . 2001;139(3):391-397. doi:10.1067/mpd.2001.116935

15. Acquazzino MA, Miller M, Myrvik M, Newby R, Scott JP. Attention Deficit Hyperactivity Disorder in Children with Sickle Cell Disease Referred for an Evaluation. J Pediatr Hematol Oncol . 2017;39(5):350-354. doi:10.1097/MPH.0000000000000847

16. Stotesbury H, Kirkham FJ, Kölbel M, et al. White matter integrity and processing speed in sickle cell anemia. Neurology . 2018;90(23):E2042-E2050. doi:10.1212/WNL.0000000000005644

17. Peterson RK, Williams S, Janzen L. Cognitive Correlates of Math Performance in School-Aged Children with Sickle Cell Disease and Silent Cerebral Infarcts. Arch Clin Neuropsychol . September 2020. doi:10.1093/arclin/acaa071

18. King AA, Strouse JJ, Rodeghier MJ, et al. Parent education and biologic factors influence on cognition in sickle cell anemia. Am J Hematol . 2014. doi:10.1002/ajh.23604

19. Knight LMJ, King AA, Strouse JJ, Tanabe P. Pediatric Neurodevelopmental Delays in Children 0 to 5 Years of Age with Sickle Cell Disease: A Systematic Literature Review. J Pediatr Hematol Oncol . 2021. doi:10.1097/MPH.0000000000002091

20. Kral MC, Brown RT, Connelly M, et al. Radiographic predictors of neurocognitive functioning in pediatric sickle cell disease. J Child Neurol . 2006;21(1):37-44. doi:10.1177/08830738060210010701

21. Power-Hays A, McGann PT. When Actions Speak Louder Than Words - Racism and Sickle Cell Disease. $N$ Engl J Med . 2020. doi:10.1056/nejmp2022125

22. Burnes DPR, Antle BJ, Williams CC, Cook L. Mothers raising children with sickle cell disease at the intersection of race, gender, and illness stigma. Heal Soc Work . 2008. doi:10.1093/hsw/33.3.211

23. Braniecki S, Moore TB, Talano J-AM, et al. Stable to Improved Neurocognitive Outcomes in Children, Adolescents \& Young Adults with High-Risk Sickle Cell Disease (SCD) Who Have Undergone Familial Haploidentical (FHI) Stem Cell Transplantation: A Prospective Study from Pre HSCT Period to 2 Years Pos. Biol Blood Marrow Transplant . 2020;26(3):S65-S66. doi:10.1016/j.bbmt.2019.12.233 
24. King AA, McKinstry RC, Wu J, et al. Functional and Radiologic Assessment of the Brain after ReducedIntensity Unrelated Donor Transplantation for Severe Sickle Cell Disease: Blood and Marrow Transplant Clinical Trials Network Study 0601. Biol Blood Marrow Transplant . 2019. doi:10.1016/j.bbmt.2019.01.008

25. Bockenmeyer J, Chamboredon E, Missud F, et al. Development of psychological and intellectual performance in transplanted sickle cell disease patients: A prospective study from pretransplant period to 5years after HSCT. Arch Pediatr . 2013. doi:10.1016/j.arcped.2013.04.012

26. Bhatnagar N, Erskine R, O'Boyle F, Chakravorty S, Roberts I, de la Fuente J. Bone Marrow Transplantation Arrests Cerebrovascular Disease Progression and Improves Psychometric Outcomes in Children with Sickle Cell Disease. Blood . 2011. doi:10.1182/blood.v118.21.11.11

27. Iannone R, Casella JF, Fuchs EJ, et al. Results of minimally toxic nonmyeloablative transplantation in patients with sickle cell anemia and $\beta$-thalassemia. Biol Blood Marrow Transplant . 2003;9(8):519-528. doi:10.1016/S1083-8791(03)00192-7

28. Pelletier W, Schulte F, Guilcher GM. Pediatric hematopoietic stem cell donors: Need for longitudinal medical and psychosocial surveillance. Pediatr Blood Cancer . 2015. doi:10.1002/pbc.25375

29. Duff K. Evidence-Based Indicators of Neuropsychological Change in the Individual Patient: Relevant Concepts and Methods | Archives of Clinical Neuropsychology | Oxford Academic.Arch Clin Neuropsychol . 2012.

30. Heilbronner RL, Sweet JJ, Attix DK, Krull KR, Henry GK, Hart RP. Official position of the American Academy of clinical neuropsychology on serial neuropsychological assessments: The utility and challenges of repeat test administrations in clinical and forensic contexts.Clin Neuropsychol . 2010. doi:10.1080/13854046.2010.526785

31. Guilmette TJ, Sweet JJ, Hebben N, et al. American Academy of Clinical Neuropsychology consensus conference statement on uniform labeling of performance test scores. Clin Neuropsychol . 2020. doi:10.1080/13854046.2020.1722244

32. Burkhardt L, Lobitz S, Koustenis E, Rueckriegel SM, Hernáiz Driever P. Cognitive and fine motor deficits in a pediatric sickle cell disease cohort of mixed ethnic origin. Ann Hematol . 2017. doi:10.1007/s00277-016$2861-1$

33. Badawy SM, Beg U, Liem RI, Chaudhury S, Thompson AA. A systematic review of quality of life in sickle cell disease and thalassemia after stem cell transplant or gene therapy. Blood Adv . 2021. doi:10.1182/bloodadvances.2020002948

34. Farrell AT, Panepinto J, Carroll CP, et al. End points for sickle cell disease clinical trials: Patient-reported outcomes, pain, and the brain. Blood Adv . 2019. doi:10.1182/bloodadvances.2019000882

\section{DATA AVAILABLITY STATEMENT}

The data that support the findings of this study are available from the corresponding author upon reasonable request. The data are not publicly available due to privacy or ethical restrictions.

\section{Hosted file}

TABLE 1 Patient Characteristics.docx available at https://authorea.com/users/457850/articles/ 554524-neuropsychological-behavioral-and-quality-of-life-outcomes-in-children-andadolescents-with-sickle-cell-disease-treated-with-nonmyeloablative-matched-siblingdonor-hematopoietic-cell-transplantation-a-case-series

\section{Hosted file}

TABLE 2 Pre and Post-HCT Neuropsychological Functioning.docx available at https:// authorea.com/users/457850/articles/554524-neuropsychological-behavioral-and-quality- 
of-life-outcomes-in-children-and-adolescents-with-sickle-cell-disease-treated-withnonmyeloablative-matched-sibling-donor-hematopoietic-cell-transplantation-a-case-series

\section{Hosted file}

TABLE 3 Pre Post-HCT Parent-Rated Attention Behavior QOL.docx available at https: //authorea.com/users/457850/articles/554524-neuropsychological-behavioral-and-qualityof-life-outcomes-in-children-and-adolescents-with-sickle-cell-disease-treated-withnonmyeloablative-matched-sibling-donor-hematopoietic-cell-transplantation-a-case-series

\section{Hosted file}

TABLE 4 Pre and Post-HCT Intellectual Functioning.docx available at https://authorea.com/ users/457850/articles/554524-neuropsychological-behavioral-and-quality-of-life-outcomesin-children-and-adolescents-with-sickle-cell-disease-treated-with-nonmyeloablativematched-sibling-donor-hematopoietic-cell-transplantation-a-case-series

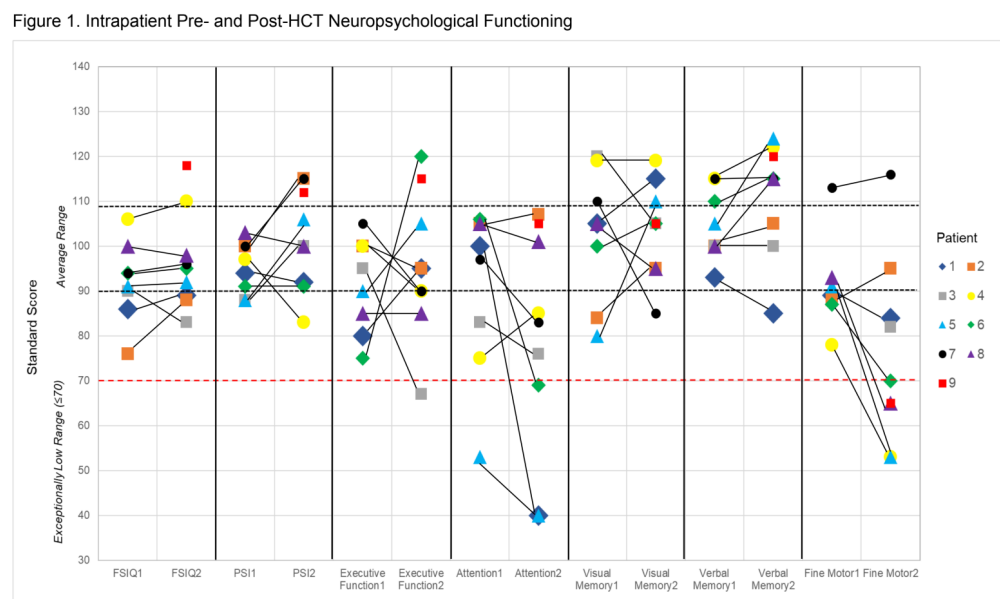


Figure 2. Correlation between Time from HCT to Assessment and Processing Speed

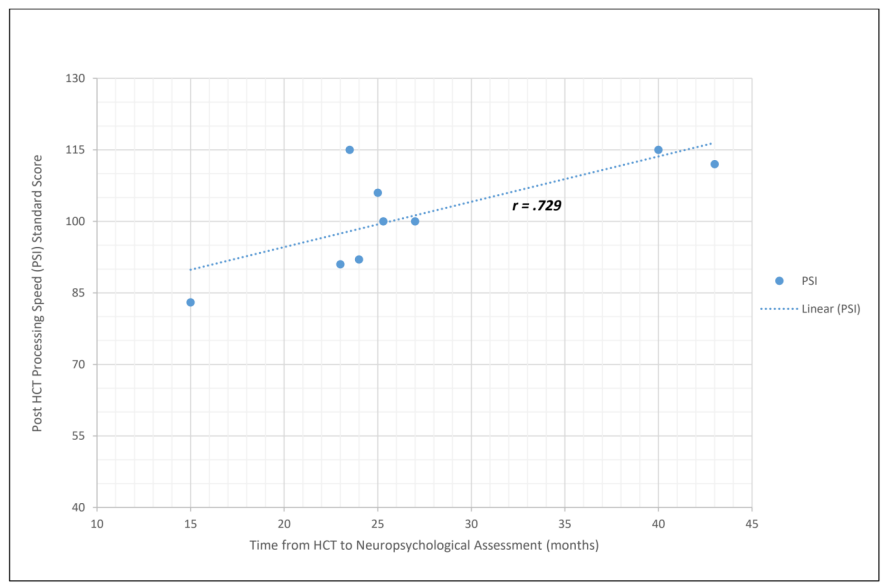

